\title{
Isolation and characterization of polyene-resistant mutants from the maize smut pathogen, $U$ stilago maydis, defective in ergosterol biosynthesis
}

\author{
Caroline S. James, Raymond S. Burden, R. S. Thomas Loeffler and John A. Hargreaves* \\ Department of Agricultural Sciences, University of Bristol, AFRC Institute of Arable Crops Research, Long Ashton \\ Research Station, Bristol BS18 9AF, UK
}

(Received 27 January 1992; revised 17 March 1992; accepted 27 March 1992)

\begin{abstract}
Ustilago maydis mutants resistant to polyene antibiotics were screened for defects in ergosterol biosynthesis. Slowgrowing mutants recovered after selecting for amphotericin B resistance were devoid of ergosterol and accumulated the methylated sterols, 14x-methylfecosterol, obtusifoliol and eburicol, indicating that these isolates were impaired in C-14 sterol demethylation and were similar to the Erg40 mutant of $U$. maydis. By contrast, nystatin- and pimaricin-resistant isolates which exhibited reduced growth rates showed a dysfunction in C-8 sterol isomerization. In these mutants (Erg2) ergosterol was replaced by the $\Delta^{8}$-sterols, ergosta-5,8,22-trienol, ergosta8,22-dienol, fecosterol and ergost-8-enol. Analysis of a random sample of polyene-resistant isolates that grew normally revealed that although most retained a typical wild-type sterol profile, two of the isolates failed to accumulate ergosterol. The major sterols detected in these isolates were ergosta-7,22-dienol and ergosta-7-enol, suggesting a lesion in C-5 sterol desaturation in these mutants (Erg3). Of four Erg2 mutants recovered, one mutant, selected on nystatin, contained low but detectable amounts of ergosterol. Ergosterol was not detected immediately after selection in the three other pimaricin-resistant Erg2 mutants. Although the growth of three of the Erg2 mutants remained unchanged during non-selective culture, one mutant reverted and began to grow at a greater rate than the rest; analysis of the sterols produced by this strain revealed that ergosterol was now present, but at lower concentrations than those in the wild-type strain. No changes in the type of sterol formed were observed in the other slower-growing Erg2 mutants even after prolonged culture. All the Erg2 mutants exhibited morphological abnormalities; sporidia were swollen and distorted, and the inability of sporidia to separate after cell division led to the development of highly branched, multicellular groups of cells.
\end{abstract}

\section{Introduction}

The biosynthesis of ergosterol and its inhibition have received considerable attention in the past, mainly due to the economic importance of ergosterol biosynthesis inhibitors (EBIs) as fungicides in agriculture and as antimycotic drugs in the treatment of fungal diseases (Baldwin, 1990; Marriott, 1990). Ergosterol is the major sterol constituent found in most fungi, although there are some exceptions (Weete, 1989). Like cholesterol in animals, and campesterol, $\beta$-sitosterol and stigmasterol in plants, ergosterol is believed to play a key role in regulating the fluidity of membranes and the activity of membrane-bound enzymes (Weete, 1989). In addition to this 'bulk' membrane function, ergosterol appears to have an additional regulatory or 'sparking' role associated with maintaining growth and cell division (Parks et

* Author for correspondence. Tel (0275) 392181; fax (0275) 394007. Abbreviation: EBI, ergosterol biosynthesis inhibitor. al., 1986; Dahl et al., 1987). The essential requirement for ergosterol in cellular and metabolic processes is demonstrated clearly by the effectiveness of EBIs in preventing fungal growth (Burden et al., 1990; Girling, 1991). However, the relative contributions of the 'bulk' membrane function and the 'sparking' role to the functioning of fungal cells remains unclear.

EBIs have proved extremely useful as tools for investigating the effect of abnormal sterol accumulation and ergosterol depletion on growth and membrane function (Burden et al., 1989). However, mutants blocked at specific points along the ergosterol biosynthetic pathway would offer a unique opportunity to study both the role of ergosterol and the mode of action of EBIs in a more precise and detailed manner. Furthermore, such mutants would be of considerable value to the molecular genetic analysis of ergosterol production and may aid the isolation of genes encoding enzymes of this pathway. 
Several sterol biosynthesis mutants have been isolated from the yeasts, Saccharomyces cerevisiae and Candida albicans (Barton et al., 1974; Karst \& Lacroute, 1977; Pesti et al., 1982; Pierce et al., 1978; Woods, 1971). Such mutants were recovered as nystatin-resistant isolates. By contrast, only one mutant deficient in ergosterol biosynthesis (Erg40) has been characterized from the maize smut pathogen, Ustilago maydis (Walsh \& Sisler, 1982). This mutant, which was deficient in C-14 sterol demethylation, was isolated by selecting for resistance to another polyene antibiotic, amphotericin B. Here we report the isolation and characterization of two additional types of polyene-resistant mutant from $U$. maydis which were unable to efficiently synthesize ergosterol because of defects in either sterol $\Delta^{8} \rightarrow \Delta^{7}$ isomerization (Erg2) or sterol $\Delta^{5(6)}$ desaturation (Erg3).

\section{Methods}

$U$. maydis strain and culture conditions. All manipulations were done using $U$. maydis isolate IMI103761. Cultures were maintained on, and sporidial suspensions prepared from YEPD medium $\left(\mathrm{g}^{-1}\right.$ : yeast extract, 10; peptone, 20; dextrose or sucrose, 20). Minimum medium was as described previously (Hargreaves \& Turner, 1989) and utilized glucose $\left(5 \mathrm{~g} \mathrm{l}^{-1}\right)$ as the carbon source. All solid media contained $20 \mathrm{~g}$ agar $1^{-1}$; polyene antibiotics were added to molten YEPD agar medium from the following stock solutions: amphotericin B (Sigma), $2 \mathrm{mg}$ (ml sterile water) ${ }^{-1}$; nystatin (Sigma), 5000 units (ml $N, N$-dimethylformamide) $)^{-1}$; and pimaricin (Sigma), $2 \cdot 5 \%(\mathrm{w} / \mathrm{v})$ aqueous suspension. All cultures were incubated at $32^{\circ} \mathrm{C}$.

Mutagenesis. Overnight $U$. maydis cultures grown in liquid YEPD medium were harvested by centrifugation $(2500 \mathrm{~g}$ for $10 \mathrm{~min})$, washed several times with sterile distilled water and then resuspended in water at a concentration of about $10^{7}$ sporidia $\mathrm{ml}^{-1}$. Samples of the sporidial suspensions $(5 \mathrm{ml})$ were irradiated for $180-220 \mathrm{~s}$ using a Phillips $30 \mathrm{~T} 8$ $90 \mathrm{~W}$ ultraviolet light placed $20 \mathrm{~cm}$ above Petri-dishes containing sporidia. The dishes were shaken continuously on a reciprocating shaker during irradiation. Between 10 and $30 \%$ of the $U$. maydis sporidia regenerated following this treatment. Mutants that appeared were suspended in $8.75 \%(\mathrm{v} / \mathrm{v})$ dimethyl sulphoxide and stored at $-70^{\circ} \mathrm{C}$.

Sterol extraction and analysis. U. maydis sporidia grown in vigorously aerated liquid YEPD medium for 16-48 $\mathrm{h}$ (depending on the growth rate of the isolate) were collected by centrifugation and washed several times with sterile water before being snap-frozen in liquid nitrogen. After freeze-drying, samples $(50 \mathrm{mg})$ were ground to a powder with sand in a mortar and pestle. Following extraction with chloroform/methanol $(2: 1, \mathrm{v} / \mathrm{v}, 30 \mathrm{ml})$ by vortexing, $\beta$-cholestanol $[1 \mathrm{mg}(\mathrm{g}$ dry weight $)^{-1}$ ] was added as an internal standard. The mixture was left to stand for several hours, decanted, and then evaporated to dryness under vacuum. The residue was dissolved in hexane and washed with saturated sodium bicarbonate solution and water. After removing hexane, the residue was dissolved in ethyl acetate and analysed on a Varian model 3700 gas chromatograph fitted with a $30 \mathrm{~m} \times 0.25 \mathrm{~mm}$ i.d. Supelco SPB-5 capillary column, using hydrogen as the carrier gas and a temperature programme of $120-265^{\circ} \mathrm{C}$ at $10^{\circ} \mathrm{C} \mathrm{min}-1$; a VGMicromass data acquisition system was used for quantification. Mass spectra (electron impact, $70 \mathrm{eV}$ ) were obtained using a Kratos MS-80 instrument coupled to the above column and helium as carrier gas.
Samples of ergosta-7,22-dienol, ergosta-8,22-dienol, ergost-7-enol and ergost-8-enol were obtained from the MRC Steroid Reference Collection (University of London); fecosterol was a gift from $\mathrm{Mr}$ A. Akers (BASF, Limburgerhof, Germany). Ergosta-5,8,22-trienol was prepared as described by Anastasia \& Fiecchi (1981), whilst ignosterol was prepared by the method of Dickson et al. (1972). All sterols used as reference samples gave the expected ${ }^{1} \mathrm{H}$ NMR spectra at $400 \mathrm{MHz}$.

\section{Results}

\section{Isolation of polyene-resistant mutants of $U$. maydis}

Following UV irradiation, $U$. maydis sporidia were plated (about $5 \times 10^{5}$ sporidia per plate) on YEPD agar medium containing amphotericin $\mathrm{B}\left(2 \mu \mathrm{g} \mathrm{ml}^{-1}\right)$, nystatin (40 units $\left.\mathrm{ml}^{-1}\right)$ or pimaricin $\left(10 \mu \mathrm{g} \mathrm{ml}^{-1}\right)$. These antibiotic concentrations inhibited the growth of sporidia for up to $4 \mathrm{~d}$. Colonies that appeared during the next $4 \mathrm{~d}$ were removed, dispersed in sterile distilled water by vortexing, and plated (about $50-100$ sporidia per plate) on YEPD medium containing no polyene antibiotic supplement. Isolates which showed reduced growth, as compared to the wild-type strain, were re-tested for polyene sensitivity and then stored for further analysis. Twelve amphotericin B-, one nystatin- and three pimaricin-resistant mutants exhibiting reduced rates of growth were recovered. These isolates represented between 1 and $5 \%$ of the total polyene-resistant colonies retrieved. A random sample of polyene-resistant isolates exhibiting near-normal growth rates were also selected for sterol analysis.

\section{Analysis of sterols accumulating in wild-type $U$. maydis strain IMI103761 and in polyene-resistant isolates}

Sterols present in the wild-type and nystatin- and pimaricin-resistant mutants were identified by comparison of their relative retention times (RRt) and mass spectra with those of authentic materials. The sterols of the amphotericin B-resistant mutants were identified by comparison with data in the literature (Ragsdale, 1975; Walsh \& Sisler, 1982). RRt and mass spectral data for all these sterols are presented in Table 1.

Ergosterol (ergosta-5,7,22-trien-3 $\beta$-ol) was the predominant sterol in the wild-type $U$. maydis strain, where it was accompanied by the closely related sterols, ergosta7,22-dienol and ergost-7-enol (Table 2). This is consistent with previous reports of the sterol composition of $U$. maydis (Ragsdale \& Sisler, 1973; Baloch et al., 1984). All the amphotericin-B-resistant isolates that exhibited reduced rates of growth contained no ergosterol. Instead, the major sterols were $14 \alpha$-methylfecosterol [14 $\alpha$-methyl$5 \alpha$-ergosta-8,24(28)-dien-3 $\beta$-ol], obtusifoliol [ $4 \alpha, 14 \alpha$-dimethyl-5 $\alpha$-ergosta-8,24(28)-dien-3 $\beta$-ol] and eburicol 
Table 1. Relative retention times (RRt) and mass spectral data of sterols identified in $U$. maydis mutants

\begin{tabular}{|c|c|c|}
\hline Sterol & $\mathrm{RRt}^{*}$ & Mass spectral data, $m / z$ (relative abundance) \\
\hline Ergosta-5,8,22-trienol & $1 \cdot 05$ & $\begin{array}{l}396\left([\mathrm{M}]^{+}, 66\right), 363(100), 337(9), 271(19), 253(19), 253(22) \text {, } \\
211(17)\end{array}$ \\
\hline Ergosta-8,22-dienol & 1.06 & $\begin{array}{l}398\left([\mathrm{M}]^{+}, 100\right), 383(38), 273(62), 271(56), 257(25), 255(32), \\
246(51), 229(62), 213(20)\end{array}$ \\
\hline Ergosterol & 1.09 & $396\left([\mathrm{M}]^{+}, 100\right), 378(11), 363(90), 271(12), 253(31), 211(19)$ \\
\hline Ergosta-7,22-dienol & $1 \cdot 11$ & $\begin{array}{l}398\left([\mathrm{M}]^{+}, 76\right), 383(18), 300(22), 271(100), 269(13), 257(13), \\
255(46), 246(28), 229(27)\end{array}$ \\
\hline ¡4 $\alpha$-Methylfecosterol & $1 \cdot 13$ & $412\left([\mathrm{M}]^{+}, 63\right), 397(100), 313(15), 245(13), 231(32), 219(20)$ \\
\hline Fecosterol & $1 \cdot 14$ & $\begin{array}{l}398([\mathrm{M}]+ \\
227(19), 213(18)\end{array}$ \\
\hline Ergost-8-enol & $1 \cdot 15$ & $400\left([\mathrm{M}]^{+}, 100\right), 385(27), 273(14), 269(15), 229(15)$ \\
\hline Ergost-7-enol & $1 \cdot 19$ & $\begin{array}{l}400\left([\mathrm{M}]^{+}, 100\right), 385(19), 273(14), 255(40), 231(11), 229(125), \\
213(12)\end{array}$ \\
\hline Obtusifoliol & $1 \cdot 21$ & $\begin{array}{l}426\left([\mathrm{M}]^{+}, 65\right), 411(100), 327(13), 259(10), 245(24), 233(12), \\
227(10)\end{array}$ \\
\hline Eburicol & $1 \cdot 38$ & $440\left([\mathrm{M}]^{+}, 70\right), 425(100), 407(24), 259(16), 241(12)$ \\
\hline
\end{tabular}

* Relative to $\beta$-cholestanol.

Table 2. Sterol composition of U. maydis wild-type and polyene-resistant mutants

The amount of each sterol is expressed as a percentage of the total free sterols. The total amount of sterols recovered was similar for all the strains analysed.

\begin{tabular}{lccccccccc}
\hline & \multicolumn{10}{c}{ Strain } \\
\cline { 2 - 9 } \multicolumn{1}{c}{ Sterol } & $\mathrm{Wt}^{*}$ & A20 & P20 & P51 & P53 & N01 & P52 & N08 & P20R $†$ \\
\hline Ergosta-5,8,22-trienol & tr & - & $7 \cdot 3$ & $11 \cdot 9$ & $8 \cdot 2$ & $15 \cdot 2$ & - & - & $16 \cdot 1$ \\
Ergosta-8,22-dienol & - & - & $20 \cdot 1$ & $19 \cdot 1$ & $16 \cdot 9$ & $27 \cdot 9$ & - & - & $22 \cdot 3$ \\
Ergosterol & $60 \cdot 5$ & - & - & - & - & $4 \cdot 0$ & - & - & $33 \cdot 4$ \\
Ergosta-7,22-dienol & $8 \cdot 2$ & - & - & - & - & - & $63 \cdot 3$ & $81 \cdot 2$ & - \\
14 $\alpha$-Methylfecosterol & - & $25 \cdot 3$ & - & - & - & - & - & - & - \\
Fecosterol & - & - & $22 \cdot 0$ & $7 \cdot 1$ & $14 \cdot 5$ & $1 \cdot 6$ & - & - & $2 \cdot 1$ \\
Ergost-8-enol & - & - & $45 \cdot 2$ & $54 \cdot 1$ & $56 \cdot 2$ & $32 \cdot 3$ & - & - & $21 \cdot 6$ \\
Ergost-7-enol & $15 \cdot 4$ & - & - & - & - & - & $23 \cdot 6$ & $13 \cdot 8$ & - \\
Obtusifoliol & - & $24 \cdot 8$ & - & - & - & - & - & - & - \\
Eburicol & tr & $33 \cdot 3$ & - & - & - & - & - & - & - \\
Unidentified sterols & $15 \cdot 9$ & $16 \cdot 6$ & $5 \cdot 5$ & $7 \cdot 8$ & $4 \cdot 2$ & $19 \cdot 0$ & $13 \cdot 1$ & $5 \cdot 0$ & $4 \cdot 5$ \\
\hline \hline
\end{tabular}

tr, Trace; -, not detected.

* Wild-type strain, IMI103761.

$\uparrow \mathrm{P} 20 \mathrm{R}$ is used to describe the reverted strain of P20 that was recovered following growth of strain P20 under non-selective conditions. Growth of the strains in YEPD or minimum medium did not affect the type of sterol formed.

$[4,4,14 \alpha$-trimethyl- $5 \alpha$-ergosta- $8,24(28)$-dien- $3 \beta$-ol]; the sterol composition of a typical amphotericin B-resistant isolate (A20) is given in Table 2. These sterols also accumulate in $U$. maydis sporidia treated with C-14 sterol demethylase inhibitors (Ragsdale \& Sisler, 1972; Ragsdale, 1975), and in the $U$. maydis Erg 40 mutant (Walsh \& Sisler, 1982). No further analyses of these isolates was done since this type of mutant had previously been isolated and characterized in detail (Walsh \& Sisler, 1982).

Analysis of free sterols from the single nystatinresistant isolate and the three pimaricin-resistant isolates that grew poorly, revealed that ergosterol was either absent (P20, P51 and P53) or present at greatly reduced amounts (N01, Table 2). All these isolates accumulated the $\Delta^{8}$ sterols, ergosta-5,8,22-trienol, ergosta-8,22dienol, fecosterol [ergosta-8,24(28)-dien-3 $\beta$-ol] and ergost-8-enol. This type of sterol also accumulates in $U$. maydis sporidia exposed to morpholine and piperidine EBIs, which are known to inhibit C-8 sterol isomerase activity (Baloch et al., 1984; Girling, 1991) and suggests that these isolates (Erg2) were defective in this step of ergosterol biosynthesis.

Analysis of sterols from polyene-resistant mutants that 


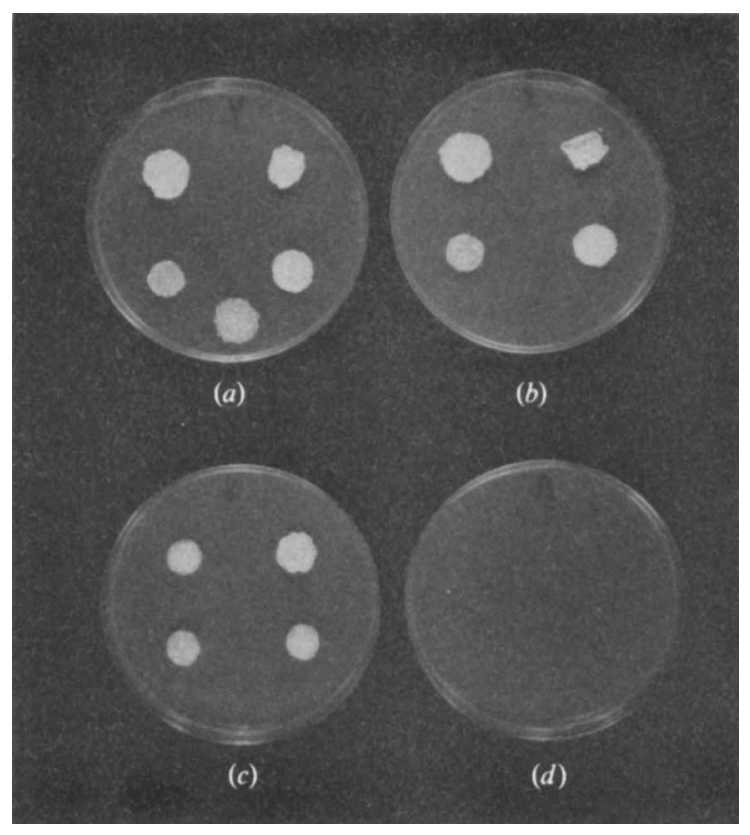

Fig. 1. Growth of $U$. maydis $\operatorname{Erg} 2$ mutants on YEPD media alone (a) or supplemented with (b) pimaricin $\left(5 \mu \mathrm{g} \mathrm{m}^{-1}\right),(c)$ nystatin (20 units $\left.\mathrm{ml}^{-1}\right)$ or $(d)$ amphotericin $\mathrm{B}\left(2 \mu \mathrm{g} \mathrm{ml}^{-1}\right)$. Each plate was inoculated with sporidia $\left(10 \mu \mathrm{l}\right.$ of a $10^{5}$ sporidia $\mathrm{ml}^{-1}$ suspension) of isolate N01 (top left-hand corner), P20 (top right-hand corner), P51 (bottom left-hand corner) and P52 (bottom right-hand corner). The wild-type $U$. maydis strain IMI103761 was inoculated at the bottom-centre of each plate.

grew normally revealed that whilst most of these isolates retained the wild-type sterol profile, two isolates recovered after selection with either nystatin (N08) or pimaricin (P52) lacked ergosterol. The major sterols which accumulated in these isolates were ergosta-7,22dienol and ergost-7-enol (Table 2) suggesting that sterol C-5 desaturation was impaired.

\section{Growth and morphology of the U. maydis Erg2 mutants}

Immediately after isolation, all four Erg2 mutants grew slowly and produced smaller colonies than those of the wild-type strain on YEPD agar plates. Addition of ergosterol $\left(80 \mu \mathrm{g} \mathrm{ml}^{-1}\right.$ in ethanol/Nonidet, $1: 1, \mathrm{v} / \mathrm{v}$; Marcireau et al., 1990) to the culture medium failed to enhance the growth of these isolates. The nystatinresistant isolate, N01, and the pimaricin-resistant isolates (P20, P51 and P53) were cross-resistant, that is they grew on media containing concentrations of pimaricin or nystatin which inhibited the growth of wild-type sporidia. By contrast, none of these mutants grew on media containing $2 \mu \mathrm{g}$ amphotericin $\mathrm{B} \mathrm{ml} \mathrm{m}^{-1}$ (Fig. 1), the concentration used to select the Erg40-like mutants.

All the Erg2 mutants exhibited morphological abnormalities. Sporidia were excessively swollen and distorted, often failing to separate after cell division. In liquid
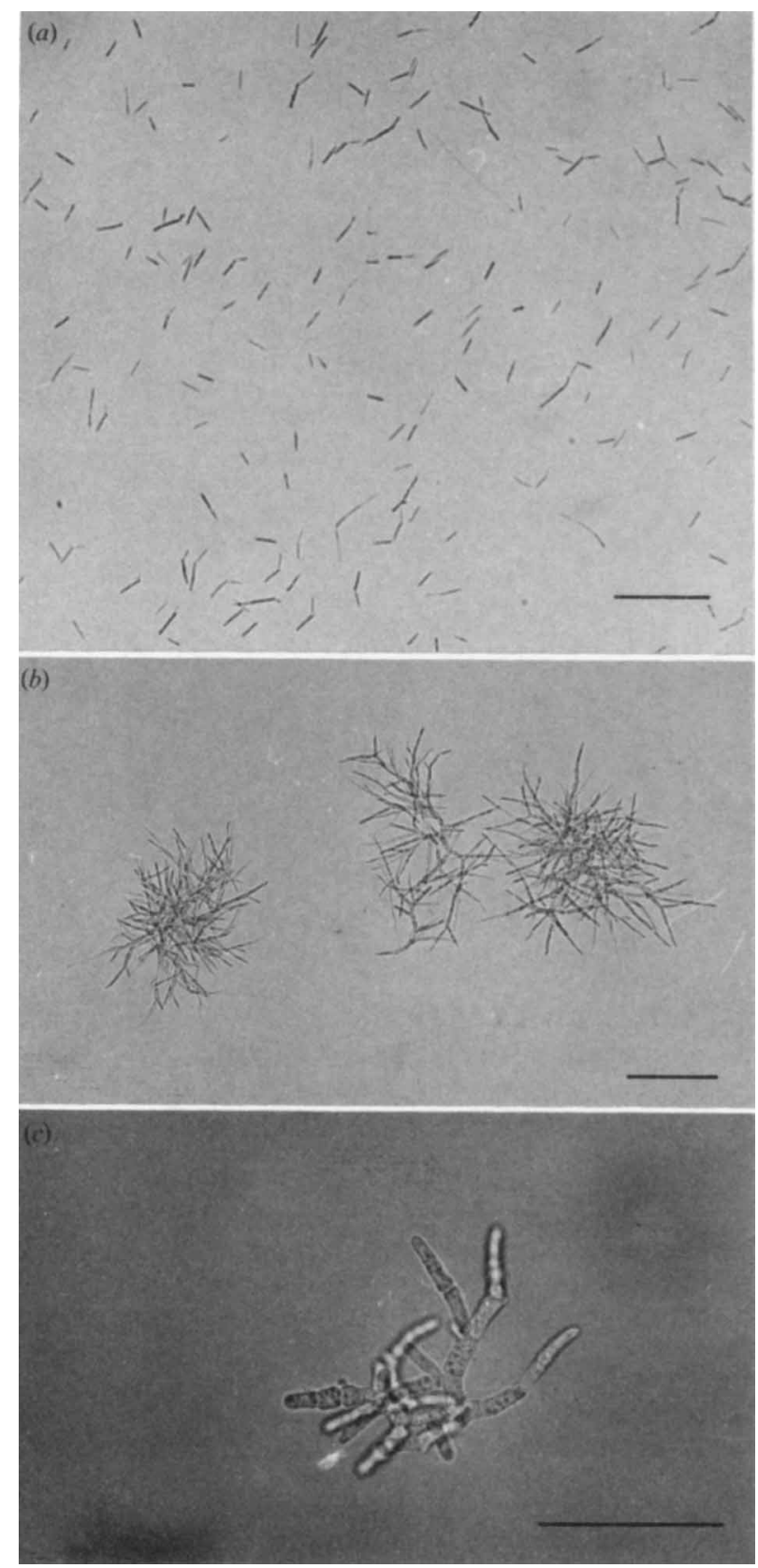

Fig. 2. Sporidia of the wild-type $U$. maydis strain IMI103761 and the Erg2 mutant P51. Wild-type sporidia grew as single isolated cells and were actively budding (a), whereas the cells of mutant P51 did not separate and formed multicellular groups $(b)$. At a higher magnification small colonies formed by the P51 isolate clearly exhibited swollen cells and a branched mode of growth $(c)$. Bars, $100 \mu \mathrm{m}(a, b), 50 \mu \mathrm{m}(c)$.

culture, this led to the formation of highly branched, multicellular groups of cells (Fig.2). Similar changes in sporidia morphology have been observed in $U$. maydis cultures exposed to the morpholine fungicide, fenpropimorph (Girling, 1991).

Three of the Erg2 mutants (N01, P51 and P53) grew at much slower rates than those of the wild-type strain in 


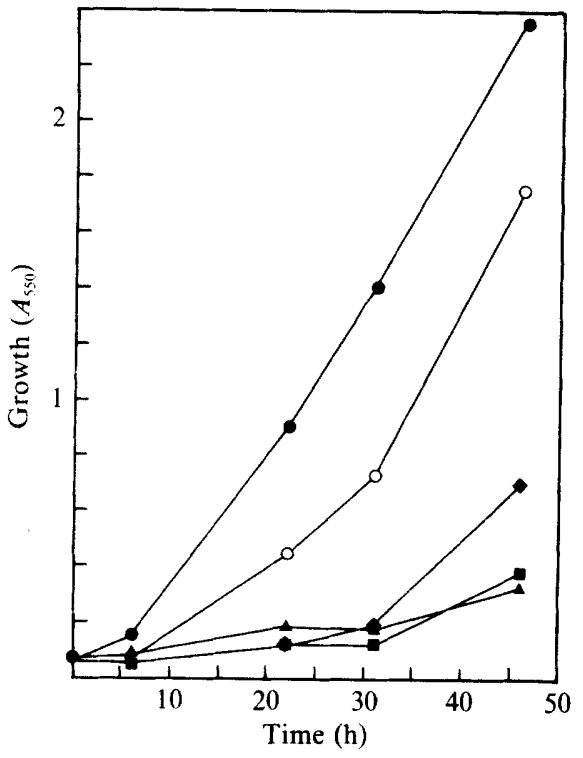

Fig. 3. Growth of the wild-type $U$. maydis strain IMI103761 (O) and of the Erg2 mutants P20 (O), P51 ( $\bullet)$, P52 $(\boldsymbol{\Delta})$ and $\mathbf{N} 01(\boldsymbol{\square})$ in liquid minimum medium. Growth was assessed by monitoring $A_{550}$.

liquid medium (Fig. 3). By contrast, one of the pimaricinresistant Erg2 mutants (P20) grew at a rate intermediate between that of the wild-type strain and the other Erg2 mutants. The majority of sporidia in this culture were essentially normal in appearance. Analysis of sterols present after $48 \mathrm{~h}$ growth revealed that, whereas N01, P51 and P53 still retained their abnormal sterol profile by accumulating $\Delta^{8}$ sterols, ergosterol was present in the isolate, P20 (P20R, Table 2), although at reduced concentrations compared to the wild-type strain IMI103761.

\section{Discussion}

Much interest has centred on the C-8 sterol isomerization step of ergosterol biosynthesis because both morpholine and related fungicides are believed to act by inhibiting this enzyme. In addition, compared to the C-14 sterol demethylation inhibitors, these compounds have shown little reduction in fungicidal effectiveness due to the development of resistance (Brent, 1988) and are, thus, suitable candidates for further development in agricultural and medical applications. The aim of the present study was to isolate a $U$. maydis mutant defective in C-8 sterol isomerization as a prelude to isolating and characterizing the ERG2 gene from $U$. maydis. Techniques for cloning and manipulating genes in $U$. maydis are highly advanced (Keon et al., 1991; Hargreaves \& Turner, 1991) and this facet makes it a useful organism for isolating and manipulating genes encoding fungicide targets (Goosey \& Moore, 1991). Furthermore, the $S$. cerevisiae $\mathrm{C}-8$ sterol isomerase gene has recently been isolated by complementation of an erg 2 mutation (Ashman et al., 1991) indicating that this strategy would be appropriate for cloning the $U$. maydis $E R G 2$ gene.

Mutants unable to produce ergosterol, because of a dysfunction in C-8 sterol isomerization, were expected to be resistant to polyene antibiotics and to be impaired in their ability to grow. The reasoning behind these assumptions came from previous work with polyeneresistant mutants of yeasts and of $U$. maydis (Barton et al., 1974; Woods, 1971; Pierce et al., 1978; Walsh \& Sisler, 1982) and from the effectiveness of C-8 sterol isomerase inhibitors in preventing growth of $U$. maydis (Baloch et al., 1984). Surprisingly, only mutants defective in C-14 sterol demethylation (Erg40) were recovered following selection with amphotericin B. By contrast, selection with either nystatin or pimaricin resulted in the recovery of Erg2 mutants which failed to produce ergosterol and accumulated $\Delta^{8}$ sterols instead. These mutants which exhibited enhanced levels of resistance to nystatin and pimaricin remained sensitive to amphotericin B, thereby, explaining why Erg2 mutants were not recovered following amphotericin $B$ selection. The reason for this sensitivity is unclear but it is possible that amphotericin B, unlike nystatin or pimaricin, can bind to $\Delta^{8}$ sterols which presumably replace ergosterol in the membranes of Erg2 mutants.

Isolates defective in C-5 sterol desaturation (Erg3) were also recovered following selection with nystatin or pimaricin. However, in contrast to the Erg2 mutants, these mutants grew at similar rates to the wild-type strain even though ergosterol was not detected. This suggested that sterols saturated in the C-5(6) position can effectively replace ergosterol in both its 'bulk' membrane and its metabolic 'sparking' role in $U$. maydis. This was, perhaps, surprising since 5-6 desaturation has been considered to be an essential requirement for 'sparking' in $S$. cerevisiae (Rodriguez \& Parks, 1983). However, our results are in agreement with the recent demonstration that the $E R G 3$ gene is not essential for either cell viability or the sparking function of sterols in $S$. cerevisiae (Arthington et al., 1991).

The rate of growth of the U. maydis Erg2 mutants was impaired and the cells produced were malformed. That $U$. maydis Erg2 mutants can survive and grow, albeit at a reduced rate, is intriguing because a presumed mode of action of morpholine fungicides is inhibition of $\mathrm{C}-8$ isomerase activity (Baloch \& Mercer, 1987). However, it has been shown that $S$. cerevisiae strains carrying an erg 2 mutation, or an $E R G 2$ gene disruption, were able to survive and grow despite the accumulation of $\Delta^{8}$ sterols (Marcireau et al., 1990; Ashman et al., 1991). In addition, it is important to emphasize that morpholine 
inhibitors are known to affect $\mathrm{C}-14$ sterol reduction as well as C-8 sterol isomerization (Baloch \& Mercer, 1984).

It is doubtful that disruption of the 'sparking' function of ergosterol contributes to the abnormal development of the Erg2 mutants since the growth of one of these isolates (N01) was impaired even though amounts of ergosterol sufficient to fulfil its sparking function accumulated. Furthermore, the 'reversion' shown by another of the Erg2 mutants (P20) demonstrated that significant amounts of ergosterol were required to restore growth and normal sporidia morphology. A similar 'reversion' has previously been observed with a C-14 demethylase mutant of $C$. albicans. This revertant also exhibited increased rates of growth during non-selective culture and accumulated detectable concentrations of ergosterol (Bard et al., 1987).

The reason why exogenously supplied ergosterol failed to influence the rate of growth of the Erg2 mutants is unclear. Previous reports have also shown that supplemental ergosterol does not restore normal growth of the $U$. maydis Erg40 mutant (Walsh \& Sisler, 1982) or of triarimol-treated $U$. maydis wild-type cells (Ragsdale \& Sisler, 1972). Thus, it would appear that $U$. maydis sporidia, unlike $S$. cerevisiae cells, are unable to take-up and utilize ergosterol from the culture medium.

Clearly $\Delta^{8}$ sterols can, at least partially, substitute for ergosterol in maintaining the structure and function of cell membranes in $U$. maydis. Nevertheless, we have encountered considerable difficulty in generating protoplasts for gene transformation from these Erg2 mutants using standard procedures (Hargreaves \& Turner, 1991). This indicated that membrane integrity was impaired by changes in sterol composition and reflected an essential role for ergosterol in maintaining membrane fluidity and function which cannot be replaced by $\Delta^{8}$ sterols. A similar diminished capacity for gene transformation, together with membrane dysfunction, has been reported for another $S$. cerevisiae ergosterol mutant (Erg6) in which C-24 sterol transmethylase activity was impaired (Gaber et al., 1989).

The abnormal sporidial morphology exhibited by the Erg2 mutants was comparable to that observed with the U. maydis Erg 40 mutant (Walsh \& Sisler 1982) and with $U$. maydis and $U$. avenae wild-type sporidia treated with the EBIs, triarimol (Ragsdale \& Sisler, 1973), tridemorph (Kerkenaar et al., 1979) and tridimefon (Buchenauer, 1977). This evidence, perhaps, points to ergosterol deficiency as the main cause of growth inhibition. Indeed, ergosterol starvation induced in $S$. cerevisiae, either by ergosterol deprivation of a sterol auxotrophic mutant (Dahl et al., 1987) or by treating cells with the morpholine fungicide, fenpropimorph (Marcireau et al., 1990), arrests growth by inhibiting bud proliferation during the $G_{1}$ phase of the cell cycle. The abnormal sporidia morphology observed with the $U$. maydis $\operatorname{Erg} 2$ mutants also indicated that the normal cell cycle process had been disrupted. However, unlike $S$. cerevisiae cells lacking ergosterol, $U$. maydis Erg2 mutants were able to divide by budding. In this case, an alternative mechanism leading to growth inhibition appears to be operating that results in the failure of mother and daughter cells to separate after cell division and which, thus, disrupts the normal pattern of sporidia differentiation.

\section{References}

ANASTASIA, M. \& FiECCHI, A. (1981). Synthesis of lichesterol [ergosta5,8(9),22-trien-3 $\beta$-ol]. Journal of the Chemical Society Perkin Transactions 1, 2125-2126.

Arthington, B. A., Bennett, L. G., Skatrud, P. L., Guynn, C. J., Barbuch, R. J., Ulbright, C. E. \& Bard, M. (1991). Cloning, disruption and sequence of the gene encoding yeast C-5 sterol desaturase. Gene 102, 39-44.

Ashman, W. H., Barbuch, R. J., Ulbright, C. E., Jarrett, H. W. \& BARD, M. (1991). Cloning and disruption of the yeast C-8 sterol isomerase gene. Lipids 26, 628-632.

BALDWIN, B. C. (1990). Inhibitors of ergosterol biosynthesis as crop protection agents. Biochemical Society Transactions 18, 61-62.

BALOCH, R. I. \& MERCER, E. I. (1987). Inhibition of sterol $\Delta^{8} \rightarrow \Delta^{7}$. isomerase and $\Delta^{14}$-reductase by fenpropimorph, tridemorph and fenpropidin in cell-free enzyme systems from Saccharomyces cerevisiae. Phytochemistry 26, 663-668.

Baloch, R. I., MerCer, E. I., Wiggins, T. E. \& Baldwin, B. C. (1984) Inhibition of ergosterol biosynthesis in Saccharomyces cerevisiae and Ustilago maydis by tridemorph, fenpropimorph and fenpropidin. Phytochemistry 23, 2219-2226.

Bard, M., Lees, N. D., Barbuch, R. J. \& Sanglard, D. (1987). Characterization of a cytochrome P450 deficient mutant of Candida albicans. Biochemical and Biophysical Research Communications 147, 794-800.

Barton, D. H. R., Corrie, J. E. T., WidDowson, D. A., Bard, M. \& WoODs, R. A. (1974). Biosynthesis of terpenes and steroids. Part IX. The sterols of some mutant yeasts and their relationship to the biosynthesis of ergosterol. Journal of the Chemical Society Perkin Transactions I 1974, 1326-1329.

Brent, K. J. (1988). Resistance experiences in Europe. In Fungicide Resistance in North America, pp. 19-27. Edited by C. J. Delph. St Paul: APS Press.

Buchenauer, H. (1977). Mode of action of tridimefon in Ustilago avenae. Pesticide Biochemistry and Physiology 7, 309-320.

Burden, R. S., COOKE, D. T. \& CARTER, G. A. (1989). Inhibitors of sterol biosynthesis and growth in plants and fungi. Phytochemistry 28, 1791-1804.

Burden, R. S., CoOke, D. T. \& Hargreaves, J. A. (1990). Mechanism of action of herbicidal and fungicidal compounds on cell membranes. Pesticide Science 30, 125-140.

Dahl, C., BiemanN, H. P. \& Dahl, J. (1987). A protein kinase antigenically related to $\mathrm{pp} 60^{\mathrm{v} \cdot \mathrm{src}}$ possibly involved in yeast cell cycle control: positive in vivo regulation by sterol. Proceedings of the National Academy of Sciences of the United States of America 84, 4012-4016.

Dickson, L. G., Patterson, G. W., Cohen, C. F. \& Dutky, S. R. (1972). Two novel steroids from inhibited Chlorella ellipsoidea. Phytochemistry 11, 3473-3477.

Gaber, R. F., Copple, D. M., Kennedy, B. K., Vidal, M. \& Bard, M. (1989). The yeast gene $E R G 6$ is required for normal membrane function but is not essential for biosynthesis of the cell-cyclesparking sterol. Molecular and Cellular Biology 9, 3447-3456. 
GIRLING, I. J. (1991). The mode of action of morpholine fungicides and their mechanisms of selectivity between fungal species. $\mathrm{PhD}$ Thesis, University of Bristol.

GoOSEY, M. W. \& MOORE, D. J. (1991). Sterol biochemistry in filamentous fungi: a coming of age. Biochemical Society Transactions 19, 769-774.

Hargreaves, J. A. \& TURNer, G. (1989). Isolation of the acetyl-CoA synthase gene from the corn smut pathogen, Ustilago maydis. Journal of General Microbiology 135, 2675-2678.

HARGREAVES, J. A. \& TURNER, G. (1991). Gene transformation in plant pathogenic fungi. In Molecular Plant Pathology - A Practical Approach, pp. 79-96. Edited by D. J. Bowles, S. J. Gurr \& M. J. McPherson. Oxford and Washington: IRL Press.

KARST, K. \& LACROUTE, F. (1977). Ergosterol biosynthesis in Saccharomyces cerevisiae, mutants deficient in the early steps of the pathway. Molecular and General Genetics 154, 269-277.

KeON, J. P. R., White, G. A. \& Hargreaves, J. A. (1991). Isolation, characterization and sequence of a gene conferring resistance to the systemic fungicide carboxin from the maize smut pathogen, Ustilago maydis. Current Genetics 19, 475-481.

Kerkenaar, A., Barug, D. \& SiJPesteijn, A. K. (1979). On the mode of action of tridemorph. Pesticide Biochemistry and Physiology 12, 195-204.

Marcireau, C., Guilloton, M. \& Karst, F. (1990). In vivo effects of fenpropimorph on the yeast Saccharomyces cerevisiae and determination of the molecular basis of its antifungal property. Antimicrobial Agents and Chemotherapy 34, 989-993.

MarRIOTT, M. S. (1990). The rational design of fungal lanosterol C14 demethylase inhibitors. In Molecular Aspects of Chemotherapy, pp. 193-204. Edited by E. Borowski \& D. Shugar. New York: Pergamon Press.
Parks, L. W., Rodriguez, R. J. \& Low, C. (1986). An essential fungal growth factor derived from ergosterol: a new end product of sterol biosynthesis? Lipids 21, 89-91.

Pesti, M., Paku, S. \& Novak, E. K. (1982). Some characteristics of nystatin-resistant sterol mutants of Candida albicans. Acta Microbiologica Academiae Scientiarum Hungaricae 29, 55-66.

Pierce, A. M., Pierce, H. D., Unrau, A. M. \& Oehlschlager, A. C. (1978). Lipid composition and polyene antibiotic resistance of Candida albicans mutants. Canadian Journal of Biochemistry 56, 135142.

Ragsdale, N. N. (1975). Specific effects of triarimol on sterol biosynthesis in Ustilago maydis. Biochimica et Biophysica Acta $\mathbf{3 8 0}$, 81-96.

RagsDale, N. N. \& Sisler, H. D. (1972). Inhibition of ergosterol synthesis in Ustilago maydis by the fungicide triarimol. Biochemical and Biophysical Research Communications 46, 2048-2054.

RAGSDALE, N. N. \& SisLER, H. D. (1973). Mode of action of triarimol in Ustilago maydis. Pesticide Biochemistry and Physiology 3, 20-29.

Rodriguez, R. J. \& PARKs, L. W. (1983). Structural and physiological features of sterols necessary to satisfy bulk membrane and sparking requirements in yeast sterol auxotrophs. Archives of Biochemistry and Biophysics 225, 861-871.

WaLSh, R. C. \& SisLer, H. D. (1982). A mutant of Ustilago maydis deficient in sterol C-14 demethylation: characteristics and sensitivity to inhibitors of ergosterol biosynthesis. Pesticide Biochemistry and Physiology 18, 122-131.

WEETE, J. D. (1989). Structure and function of sterols in fungi. Advances in Lipid Research 23, 115-167.

Woods, R. A. (1971). Nystatin-resistant mutants of yeast: alterations in sterol content. Journal of Bacteriology 108, 69-73. 